\title{
Comparison of treatment outcomes in severe personality disorder patients with or without substance use disorders: a 36-month prospective pragmatic follow-up study
}

\author{
This article was published in the following Dove Press journal: \\ Neuropsychiatric Disease and Treatment \\ 21 June 2016 \\ Number of times this article has been viewed
}

\author{
Fernando Lana ${ }^{1-3}$ \\ Carmen Sánchez-Gill ${ }^{1-3}$ \\ Núria D Adroher 4,5 \\ Víctor Pérez ${ }^{1-4}$ \\ Guillem Feixas ${ }^{6}$ \\ Josep Martí-Bonany ${ }^{1-3}$ \\ Marta Torrens ${ }^{1-4}$ \\ 'Institute of Neuropsychiatry and \\ Addictions (INAD), Centre Emili \\ Mira and Hospital del Mar, Parc de \\ Salut Mar, Barcelona, Spain; ${ }^{2}$ Mental \\ Health Research Networking \\ Center (CIBERSAM), Madrid, \\ Spain; ${ }^{3}$ Department of Psychiatry, \\ Autonomous University of Barcelona, \\ Barcelona, Spain; ${ }^{4}$ IMIM (Hospital \\ del Mar Medical Research Institute), \\ Barcelona, Spain; ${ }^{5}$ Public Health and \\ Epidemiology Research Networking \\ Center (CIBERESP), Madrid, Spain; \\ ${ }^{6}$ Department of Clinical Psychology \\ and Psychobiology, Faculty of \\ Psychology, University of Barcelona, \\ Barcelona, Spain
}

Background: Concurrent personality disorder (PD) and substance use disorder (SUD) are common in clinical practice. However, SUD is the main criterion for study exclusion in most psychotherapeutic studies of PD. As a result, data on treatment outcomes in patients with concurrent PD/SUD are scarce.

Methods: The study sample consisted of 51 patients diagnosed with severe PD and admitted for psychotherapeutic treatment as a part of routine mental health care. All patients were diagnosed with PD according to the Structured Clinical Interview for PD. Patients were further assessed (DSM-IV diagnostic criteria) to check for the presence of concurrent SUD, with 28 patients diagnosed with both disorders (PD-SUD). These 28 cases were then compared to the 23 patients without SUD (PD-nSUD) in terms of psychiatric hospitalizations and psychiatric emergency room (ER) visits before and during the 6-month therapeutic intervention and every 6 months thereafter for a total of 36 months.

Results: The baseline clinical characteristics correspond to a sample of PD patients $(78 \%$ met $D S M-I V$ criteria for borderline PD) with poor general functioning and a high prevalence of suicide attempts and self-harm behaviors. Altogether, the five outcome variables - the proportion and the number of psychiatric inpatient admissions, the number of days hospitalized, and the proportion and the number of psychiatric ER visits - improved significantly during the treatment period, and this improvement was maintained throughout the follow-up period. Although PD-SUD patients had more psychiatric hospitalizations and ER visits than PD-nSUD patients during follow-up, the differences between these two groups remained stable over the study period indicating that the treatment was equally effective in both groups.

Conclusion: Specialized psychotherapy for severe PD can be effectively applied in patients with concurrent PD-SUD under usual practice conditions. These findings suggest that exclusion of patients with dual disorders from specialized treatments is unjustified.

Keywords: personality disorder, substance use disorder, borderline personality disorder, dual disorders, psychotherapy, pragmatic clinical study

\section{Background}

(ander Fernando Lan Couropsychiatry and Addictions (INAD), Emili Mira Center, Parc de Salut Mar de Barcelona, c/Prat de la Riba, I7I Santa Coloma de Gramenet, Barcelona ES-0892I, Spain

Tel +34934628900

Fax +3493468 3742

Email 25018@parcdesalutmar.cat
Numerous studies have shown that the co-occurrence of personality disorders (PDs) with substance use disorders (SUDs) is high but variable in both the general population it is estimated that from $29 \%$ to $48 \%$ also have PD. Conversely, among individuals in the general population with PD, $7 \%-16 \%$ are believed to have concurrent SUD. ${ }^{1}$ and in clinical populations. Among individuals in the general population with SUD, 
By contrast, 34\%-91\% of treatment-seeking patients with SUD also have PD, and up to $84 \%$ of patients seeking treatment for PD also have concurrent SUD..$^{2-5}$ The co-occurrence of PD with SUD, especially when impulsivity predominates, negatively impacts the clinical course and outcomes of SUD. ${ }^{3,6-8}$ Moreover, some evidence suggests that concurrent SUD may render PD psychopathology more serious, although the evidence is not as strong. ${ }^{5}$

Studies that have evaluated the efficacy of psychotherapy in patients with SUD and comorbid PD $^{9-11}$ are sparse and often controversial. ${ }^{12,13}$ In contrast, a variety of different psychotherapeutic interventions have been proven effective for PD in several prospective studies, ${ }^{14-16}$ and similar outcomes are achieved regardless of whether the interventions are applied in outpatient settings, ${ }^{17-21}$ day hospital programs, ${ }^{22-27}$ or inpatient programs. ${ }^{28}$ However, many of the studies that have investigated the efficacy of PD psychotherapy have excluded PD patients with concurrent SUD (PD-SUD). ${ }^{18,20,25,29}$ In other studies, not all patients with dual PD-SUD were excluded from specialized treatments, although the proportion of patients excluded can be highly variable. More restrictive studies exclude 70\%-94\% of PDSUD patients, ${ }^{17,21,22,24}$ while less restrictive studies exclude less than $50 \%$ of the PD-SUD patients. ${ }^{19,26,27,30}$

Overall, the available evidence ${ }^{14,31,32}$ indicates that SUD is a barrier for access to specialized PD treatment. PD-SUD patients are typically excluded for both methodological ${ }^{31}$ and clinical ${ }^{14}$ reasons, a phenomenon that is evident in randomized controlled trials (RCT), which are often designed to increase study feasibility and to decrease response heterogeneity. However, by applying highly restrictive eligibility criteria, such studies may yield results whose external validity is low, due in part to sample bias. The same is true for PD psychotherapy studies, in which SUD is the leading criterion for study exclusion. ${ }^{31}$ Such studies tend to recruit "pure" rather than "typical" patients and, as a consequence, to perpetuate the gap between research and clinical practice. ${ }^{31,32}$ Patients with concurrent PD-SUD are also excluded because clinical studies have shown ${ }^{7,14}$ that such patients can be problematic, with a high dropout rate and a greater propensity to act violently (both in self-harm and acts of aggression against others). All of these factors could explain the tendency among professionals to refer these patients elsewhere. ${ }^{14}$ Nevertheless, several studies ${ }^{33-35}$ have shown that standard dialectical behavior therapy (DBT) can be effectively applied to patients with borderline PD (BPD) with or without comorbid SUD. Consequently, the exclusion of comorbid patients from specialized treatment may not be justified.
In a previous pragmatic study, ${ }^{27}$ we assessed a 6-month psychotherapeutic treatment for severe PD (mainly [78\%] BPD patients) in a sample of patients with poor general functioning and a high prevalence of suicide attempts, self-harm behaviors, and comorbid SUD. In that study, we used a multicomponent integrated psychotherapeutic approach that combines effective techniques from various therapeutic models, primarily DBT and mentalization-based treatment (MBT). We found that this combined treatment was effective in preventing psychiatric inpatient readmissions and repeated psychiatric emergency room (ER) visits during the 6-month treatment period, a benefit that was maintained during the entire 36-month follow-up.

Given the scarcity of data on treatment outcomes in patients with PD-SUD, we carried out the present study to examine the following research questions: 1) Can a psychotherapeutic program for patients with PD without SUD (PD-nSUD) be effectively applied to those with comorbid SUD (PD-SUD)? 2) Are there significant differences in treatment outcome between PD patients with and without SUD?

\section{Materials and methods Study and design}

This study consists of a secondary analysis of data obtained in a previous pragmatic study. ${ }^{27}$ We carried out a pre-post, prospective, pragmatic study of patients admitted to an integrated therapeutic program for PD with serious psychiatric symptomatology and low psychosocial functioning (severe PD). The study was conducted at a day hospital within the Barcelonès-Nord mental health network, a public health care catchment area that provides services to a population of approximately 299,000 inhabitants in the metropolitan area of Barcelona. Private practice in this area is scarce and limited to the outpatient setting. According to guidelines provided by the local health authorities, medical-psychiatric health care is sectorized, and patients should be referred to the appropriate hospital for their sector. Consequently, nearly all subjects with mental health disorders are referred to the only sectorial psychiatric hospital within the Barcelonès-Nord mental health network. The study was approved by the local Ethics Committee (Comité Etico de Investigación Clínica del Centre Emili Mira-Hospital Universitari Germans Trias i Pujol), and all patients signed an Informed Consent Form.

\section{Participants and procedure}

The sample was selected from all consecutive referrals to the severe PD program from September 2001 to December 2006 , inclusive. The inclusion criteria for admittance to the program were the following: 1) DSM-IV criteria $^{36}$ for BPD; 
or 2) $D S M-I V$ criteria $^{36}$ for another PD together with selfinflicted lesions, suicidal behavior, or impulsive behavior ( $\geq$ two fields: spending, sex, substance abuse, reckless driving, and food binges) of the fourth $D S M-I V$ criteria for BPD; 3) a score of 50 or less in the Global Assessment of Functioning (GAF) scale of $D S M-I V ;{ }^{36}$ and 4 ) ability to initiate treatment without having to take a leave from studies or work. The exclusion criteria were as follows: presence of $D S M-I V^{36}$ criteria for 1) schizophrenia, 2) bipolar I disorder, 3) mental retardation, or 4) severe maladaptive behaviors associated with SUD that interfered seriously with treatment (eg, either highly aggressive behaviors such as frequent physical fights, or excessive time dedicated to SUD-related activities [such as obtaining, using, and/or recovering from the effects of the substance] such that attendance at the pretreatment assessment meetings was compromised). All patients were referred by a trained therapist after assessment involving a clinical interview performed in accordance with the $D S M-I V$ criteria. ${ }^{36}$ The assessment protocol included administration of the following: a sociodemographic survey, a psychiatric service use registry, a substance use questionnaire, drug urinalysis screening (cannabis, opiates, cocaine, amphetamine, LSD, and benzodiazepines), the DSM-IV GAF scale, and the Spanish version of the Structured Clinical Interview for DSM-IV Personality Disorders (SCID-II). ${ }^{37}$ Clinical severity was evaluated pragmatically according to the occurrence of crisis events, a composite of suicidal and self-harming behaviors and hospitalization, proposed by Bateman and Fonagy. ${ }^{19}$ These authors included hospital admission because patients are offered inpatient care in anticipation of suicide attempts and severe self-harm. We assessed when a patient presented one, two, or three of these events. Transient psychotic symptoms were assessed by clinical interview and the paranoid ideation criteria of the fourth $D S M-I V$ for BPD.

The flow of participants through the study is shown in Figure 1. Of the patients assessed during the designated period, three were excluded because they met criteria for a diagnosis of schizophrenia (two cases) or bipolar I

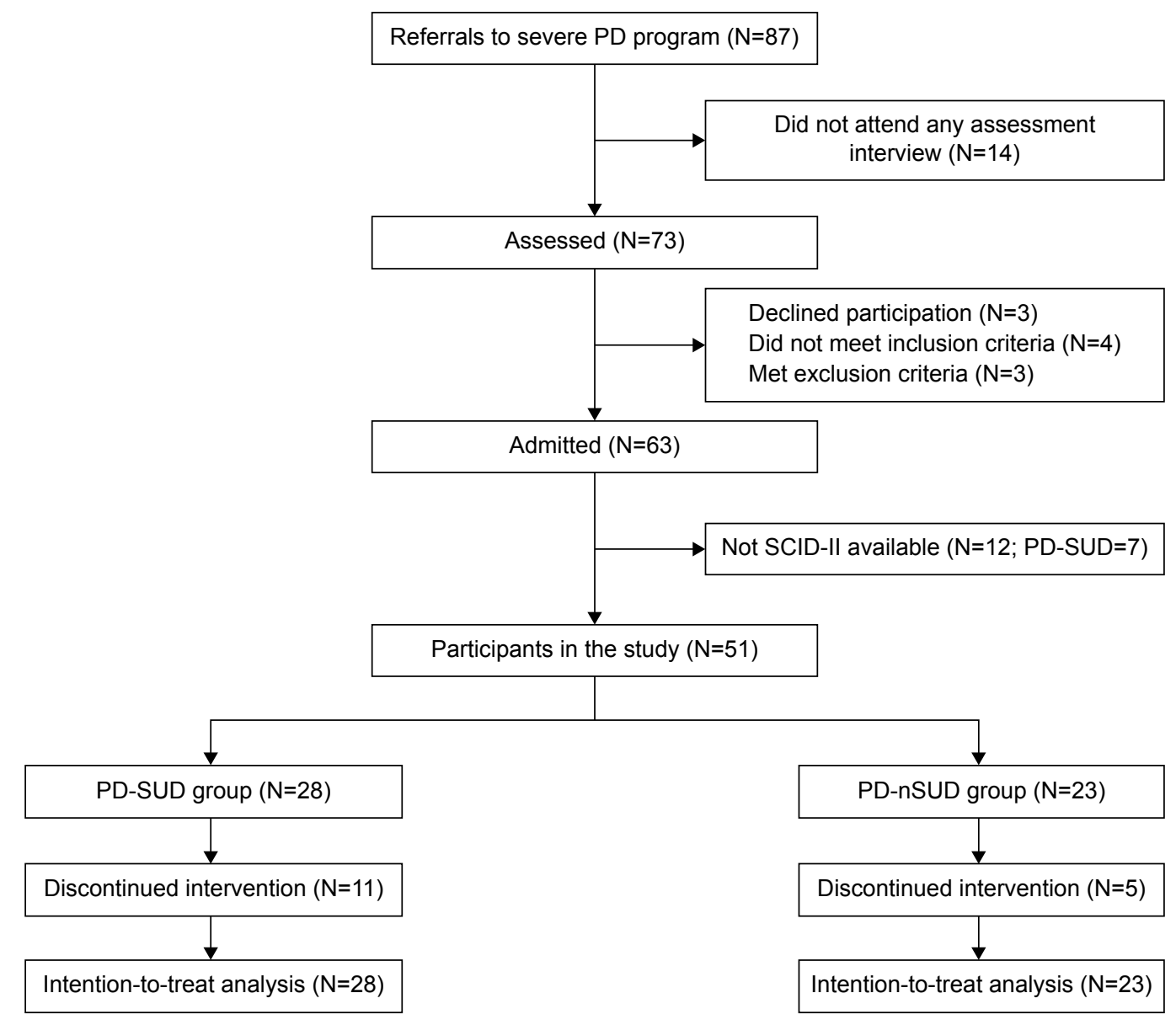

Figure I Flow of participants in the study.

Abbreviations: SCID-II, Structured Clinical Interview for DSM-IV personality disorders; PD, personality disorder; PD-SUD, personality disorder with comorbid substance use disorder; PD-nSUD, personality disorder without substance use disorder. 
disorder criteria (one patient). No patients were excluded for criterion 4 of the exclusion criteria. In 12 cases (admitted in 2001 and 2002), it was not possible to administer the SCID-II because of problems related to the program launch. Thus, the final sample included 51 patients diagnosed with PD.

The SUD group was defined according to the following criteria: 1) DSM-IV criteria $^{36}$ for SUD (alcohol, cannabis, opiates, amphetamine, LSD, or cocaine). Notably, benzodiazepines, nicotine, and caffeine were not included because these SUD are not generally excluded in psychotherapy efficacy studies and 2) at least one positive drug urinalysis screening for cannabis, opiates, amphetamine, LSD, or cocaine in the last 3 months (alcohol use was not considered). In the final sample, 28 cases $(54.9 \%)$ were included in the PD-SUD group and $23(45.1 \%)$ in the PD-nSUD group. Sixteen patients $(31.4 \%)$ of the total sample prematurely dropped out of the program: eleven (39.3\%) in the PD-SUD group and five $(21.7 \%)$ in the PD-nSUD group. However, because the dropout rate did not differ significantly between the two groups $\left(\chi^{2}=1.08 ; d f=1\right.$; $P=0.30$ ), the patients who abandoned the program were included in the analysis. During the treatment period, one patient (PD-nSUD group) died as a result of self-inflicted lesions, and 17 months after finishing treatment, one patient (PD-SUD group) committed suicide; in both cases, the last observations were carried forward.

\section{Intervention: the integrated therapeutic program for severe PD}

The treatment program, which has been described previously in detail, ${ }^{27}$ is a multicomponent, integrated treatment that adopts the four general therapeutic strategies suggested by Livesley ${ }^{38}$ as a general organizational framework and combines effective techniques from various evidence-based therapeutic models, such as $\mathrm{DBT}^{39}$ and MBT. ${ }^{40}$ The program offers 6 months of treatment, open to both male and female subjects aged $18-55$ years. This is an open therapeutic program; however, new patients are generally not allowed to join the program during the February to September time period to assure that the treatment program can be finalized either before (preferably) or during the month of July (members of the treatment team have a part-time dedication to the program and there are strict limitations with regard to vacation time during the treatment period). All patients must have a confirmed diagnosis of a PD (usually BPD). Most patients have low general functioning and the presence of serious behavioral disorders, most commonly suicidal behavior and self-inflicted lesions. Before and after discharge, patients receive standard psychiatric treatment. The program, which takes place from Monday to Friday, comprises several weekly group interventions: 1) skills training (2.5 hours) based on DBT techniques; ${ }^{39}$ 2) relationship therapy (1.5 hours), supported by MBT; ${ }^{40} 3$ ) stress management ( 2 hours); and 4) psychoeducational therapy (1.5 hours). In addition, the program includes the following: 5) individual therapy once a week, consisting of support psychodynamic psychotherapy or DBT, depending on the therapist's approach; 6) medication review; 7) nursing consultation; and 8) telephone consultation (from Monday to Friday, from 9:00 am to 4:00 pm). Group psychotherapy is conducted in cotherapy by nursing staff and/ or psychotherapists. Individual psychotherapy is conducted by a team of four psychotherapists with more than 10 years of training and extensive psychotherapeutic experience at public hospitals. Medication is based on a symptom-targeted approach $^{41}$ and is prescribed by a psychiatrist from the treatment team. Pharmacotherapy prioritizes antidepressant, mood stabilizers, and antipsychotic drugs; polypharmacy and benzodiazepines are discouraged. Once a week, the whole team holds a supervisory meeting ( 1.5 hours) to discuss the cases and the program.

\section{Outcome variables}

The main outcome variable was the need for psychiatric hospitalization, assessed in several ways: 1) as a proportion of psychiatric inpatient admissions treated as a dichotomous variable (presence or absence of admissions); 2) as a count variable (number of admissions); and 3 ) as a continuous variable (days of hospitalization). Other outcome variables were 4) proportion of ER visits for psychiatric care, also assessed as a dichotomous variable (presence or absence of psychiatric ER visits) and 5) number of ER visits. Assessments were made before admission to the program or baseline assessment $\left(\mathrm{T}_{0}\right.$, including the 6 months prior to starting treatment), after 6 months of treatment $\left(\mathrm{T}_{1}\right)$, and every 6 months until completion of the 36-month follow-up $\left(\mathrm{T}_{2}\right.$, including months $7-12$ from starting treatment; $\mathrm{T}_{3}$, months 13-18; $\mathrm{T}_{4}$, months 19-24; $\mathrm{T}_{5}$, months 25-30; $\mathrm{T}_{6}$, months 31-36). We searched the psychiatric hospital database (the only such database within the mental health network) to obtain information about hospitalizations and ER visits among the study sample before and during treatment and throughout the follow-up period. It is possible (although unusual) that a patient could have been treated at another hospital outside of our catchment area. Therefore, as a part of routine mental health assessment, patients were asked at the baseline interview about the use of psychiatric services, clinical report forms were requested, and, if appropriate, the data were verified with the mental health care professionals who treated the patient previously. Similarly, again as part 
of routine procedures, during treatment at the day hospital, patients and their families were asked about the use of psychiatric services. Finally, throughout the follow-up period, the professionals who treated the patients were asked about the use of psychiatric services by these patients; data were verified against the patient medical records.

\section{Statistical analyses}

The analyses were performed with the SPSS ${ }^{\circledR}$ statistics program (SPSS version 21.0; IBM Corporation, Armonk, NY, USA) and the R statistical software (version 3.1.2; https:// www.r-project.org/, The R Foundation, Vienna, Austria). Count and continuous variables were described by mean values and standard deviations, and categorical variables were defined by absolute frequencies and percentages, all of which were calculated for each group (PD-SUD vs PD-nSUD). Baseline characteristics were compared using chi-square statistics with Yates correction for dichotomous variables, the Mann-Whitney $U$-test for count variables, and the Student's $t$-test for continuous variables after comparing the variances between the two samples. All outcome variables were analyzed on an intention-to-treat analysis. Values of $P<0.05$ were considered significant. To quantify the difference between the mean values of continuous outcome variables, the effect size was calculated as the difference between the baseline mean and the last mean $\left(\mathrm{T}_{6}\right)$, divided by the baseline standard deviation. ${ }^{42}$

For the analysis of change throughout the follow-up, generalized estimating equation (GEE) was used, a procedure that goes beyond the generalized linear model for the analysis of repeated measures. GEE takes into account that the same patients are repeatedly measured and uses all the available data, irrespective of the number of repeated measurements. ${ }^{43}$ We performed a GEE analysis with each of the outcome measures as dependent variables and time (continuous), SUD, and the interaction time $\times$ SUD as independent variables. Therefore, GEE logistic (outcomes 1 and 4), Poisson (outcomes 2 and 5), and Gaussian (outcome 3) regressions were performed. To reduce variances attributed to nonrelevant factors, age and sex were included as covariates. A likelihood ratio test was applied to determine whether the model fitted better when interactions were included in the model.

\section{Results \\ Sample description}

Differences between sociodemographic characteristics were not significant (Table 1) except for the fact that the PD-SUD

Table I Baseline demographic and clinical characteristics

\begin{tabular}{|c|c|c|c|c|c|c|}
\hline \multirow[t]{2}{*}{ Variable } & \multicolumn{2}{|c|}{ PD-SUD (n=28) } & \multicolumn{2}{|c|}{ PD-nSUD (n=23) } & \multirow[t]{2}{*}{$\chi^{2}$} & \multirow[t]{2}{*}{ P-value } \\
\hline & $\mathbf{n}$ & $\%$ & $\mathbf{n}$ & $\%$ & & \\
\hline Female & 16 & 57.1 & 15 & 65.2 & 0.09 & 0.77 \\
\hline Never married & 17 & 60.7 & II & 47.8 & 0.41 & 0.52 \\
\hline Living with family of origin & 22 & 78.6 & 12 & 52.2 & 2.86 & 0.10 \\
\hline Less than high school (education) & 15 & 53.6 & 15 & 65.2 & 0.31 & 0.58 \\
\hline Statutory sick/disability pension & 13 & 46.4 & 14 & 60.9 & 0.56 & 0.45 \\
\hline Suicide attempts & 23 & 82.1 & 18 & 78.3 & 0.00 & 1.00 \\
\hline Self-harming behavior & 17 & 60.7 & 16 & 69.6 & 0.13 & 0.72 \\
\hline Aggression episodes & 12 & 42.9 & 4 & 17.4 & 2.71 & 0.10 \\
\hline Transient psychotic symptoms & 15 & 53.6 & 8 & 34.8 & 1.12 & 0.29 \\
\hline Any mood disorder & 25 & 89.3 & 20 & 87.0 & 0.00 & 1.00 \\
\hline Any anxiety disorder & 19 & 67.9 & 16 & 69.6 & 0.00 & 1.00 \\
\hline Borderline personality disorder & 23 & 82.1 & 17 & 73.9 & 0.14 & 0.71 \\
\hline Psychiatric inpatient admission $\left(\mathrm{T}_{0}\right)$ & 22 & 78.6 & 10 & 43.5 & 5.24 & 0.02 \\
\hline \multirow[t]{2}{*}{ Psychiatric ER visit $\left(\mathrm{T}_{0}\right)$} & 22 & 78.6 & 9 & 39.1 & 6.67 & 0.01 \\
\hline & Mean & SD & Mean & SD & $t$ or $z$ & P-value \\
\hline Age at start of treatment & 30.5 & 6.8 & 36.9 & 10.7 & -2.47 & 0.02 \\
\hline Age at first psychiatric inpatient admission & 25.6 & 5.7 & 30.8 & 10.6 & -1.83 & 0.08 \\
\hline Age at first psychiatric consultation & 19.6 & 8.9 & 20.3 & 10.5 & -0.26 & 0.80 \\
\hline Time since first consultation, years & 10.9 & 7.2 & 16.5 & 10.1 & -2.26 & 0.03 \\
\hline GAF scale & 37.9 & 4.2 & 40.9 & 4.2 & -2.53 & 0.02 \\
\hline Number of psychiatric admissions $\left(\mathrm{T}_{0}\right)$ & 1.7 & 1.4 & 0.6 & 0.9 & 3.09 & 0.00 \\
\hline Days of psychiatric hospitalization $\left(\mathrm{T}_{0}\right)$ & 35.9 & 31.9 & 13.7 & 21.2 & 2.97 & 0.00 \\
\hline Number of psychiatric ER visits $\left(\mathrm{T}_{0}\right)$ & 2.5 & 2.3 & 0.8 & 1.3 & 3.18 & 0.00 \\
\hline
\end{tabular}

Abbreviations: GAF, Global Assessment of Functioning; PD-SUD, personality disorder with comorbid substance use disorder; PD-nSUD, personality disorder without substance use disorder; SD, standard deviation; ER, emergency room. 
group was younger. Similarly, PD-SUD patients were also younger at the first psychiatric admission and had more previous psychiatric hospitalizations and psychiatric ER visits. All patients reported at least one crisis event (suicidal or self-harming behavior, or hospitalization). Fourteen patients $(50.0 \%)$ in the PD-SUD group and five patients $(21.7 \%)$ in the PD-nSUD group $\left(\chi^{2}=3.19 ; d f=1 ; P=0.07\right)$ reported three crisis events, and nine patients $(32.1 \%)$ in the PD-SUD group and 12 patients $(52.2 \%)$ in the PD-nSUD group $\left(\chi^{2}=1.35 ; d f=1\right.$; $P=0.25)$ reported two crisis events. Although the PD-SUD group presented more behavioral disorders, transient psychotic symptoms, and a higher proportion of BPD diagnoses, these differences were not significant. However, the PD-SUD group had a significantly lower GAF score (Table 1). At baseline, 28 patients $(100 \%)$ in the PD-SUD group and 22 patients $(95.7 \%)$ in the PD-nSUD group $\left(\chi^{2}=0.01 ; d f=1 ; P=0.92\right)$ reported that they were taking psychotropic medications. SUD consisted of the following use disorders: alcohol (20 patients; 71.4\%), cannabis (19 patients; 67.9\%), cocaine (16 patients; $57.1 \%$ ), amphetamine (four patients; 14.3\%), and opioid (three patients; 10.7\%). In addition, 19 patients (67.9\%) used two or more substances; eleven patients $(39.3 \%)$ met criteria for dependence on at least one substance.

\section{Outcome variables}

There was a significant reduction $(P<0.001)$ across time (Table 2) in the five outcome variables (the proportion and the number of psychiatric inpatient admissions, days of hospitalization, and the proportion and the number of psychiatric ER visits). This indicates that the treatment was effective in reducing psychiatric hospitalizations and psychiatric ER visits, a benefit that was maintained during the entire 36-month follow-up. Comparatively (Table 2), PD-SUD patients suffered more psychiatric hospitalizations and ER visits than PD-nSUD patients during the 36-month follow-up (Figures 2 and 3). However, the differences between these two groups were stable over time as shown by the interaction time $\times$ SUD (Table 2), which was not significant in four of the outcome variables: the proportion of psychiatric inpatient admissions, number of psychiatric inpatient admissions, proportion of psychiatric ER visits, and number of psychiatric ER visits. These findings show that the treatment was equally effective in both groups as measured by these specific outcome variables. Conversely, the interaction time $\times$ SUD (Table 2) differed significantly for the number of days of hospitalization, as the difference between the two groups decreased significantly over time, meaning that the treatment was more effective for the PD-SUD patients in which the duration of hospitalization decreased significantly. The effect size for the PD-SUD group was higher $(d=0.94)$ than that for the PD-nSUD group $(d=0.62)$.

\section{Discussion}

In this study, our main aim was to determine treatment outcomes in patients with concurrent SUD and PD who underwent a multicomponent treatment approach that integrates

Table 2 Outcome variables: analysis of change throughout the 36-month follow-up

\begin{tabular}{|c|c|c|c|c|c|c|c|c|c|}
\hline \multicolumn{10}{|c|}{ Psychiatric hospitalization } \\
\hline & \multicolumn{3}{|c|}{ Inpatients (proportion of admissions) } & \multicolumn{3}{|c|}{ Number of admissions } & \multicolumn{3}{|c|}{ Days of hospitalization } \\
\hline & B (SE) & $P$-value & OR $(95 \% \mathrm{Cl})$ & B (SE) & $P$-value & $95 \% \mathrm{Cl}$ of $\mathrm{B}$ & B (SE) & $P$-value & $95 \% \mathrm{Cl}$ of $\mathrm{B}$ \\
\hline (Intercept) & $-0.57(0.99)$ & 0.56 & $0.56(0.08 / 3.91)$ & $-1.32(0.75)$ & 0.08 & $-2.79 / 0.14$ & $9.62(5.24)$ & 0.07 & $-0.65 / 19.90$ \\
\hline Time & $-0.32(0.07)$ & 0.00 & $0.73(0.63 / 0.84)$ & $-0.24(0.07)$ & 0.00 & $-0.38 /-0.11$ & $-1.28(0.45)$ & 0.00 & $-2.16 /-0.40$ \\
\hline SUD & $1.03(0.42)$ & 0.02 & $2.81(1.22 / 6.45)$ & $1.08(0.33)$ & 0.00 & $0.43 / 1.73$ & $14.18(4.28)$ & 0.00 & $5.79 / 22.56$ \\
\hline Sex & $0.83(0.37)$ & 0.03 & $2.28(1.10 / 4.75)$ & $0.66(0.29)$ & 0.02 & $0.10 / 1.22$ & $5.84(2.63)$ & 0.03 & $0.68 / 10.99$ \\
\hline Age & $-0.03(0.03)$ & 0.32 & $0.97(0.93 / 1.03)$ & $-0.00(0.02)$ & 0.86 & $-0.04 / 0.04$ & $-0.16(0.13)$ & 0.21 & $-0.40 / 0.09$ \\
\hline Time:SUD & $-0.01(0.16)$ & 0.97 & $0.99(0.73 / 1.35)$ & $-0.01(0.16)$ & 0.97 & $-0.3 \mathrm{I} / 0.30$ & $-2.15(0.76)$ & 0.00 & $-3.64 /-0.67$ \\
\hline \multicolumn{10}{|c|}{ Psychiatric emergency room visit } \\
\hline & \multicolumn{5}{|c|}{ Patients (proportion of ER visits) } & \multicolumn{3}{|c|}{ Number of ER visits } & \\
\hline & \multicolumn{2}{|c|}{ B (SE) } & $P$-value & \multicolumn{2}{|c|}{ OR $(95 \% \mathrm{Cl})$} & B (SE) & \multicolumn{2}{|c|}{$P$-value } & $95 \% \mathrm{Cl}$ of $\mathrm{B}$ \\
\hline (Intercept) & \multicolumn{2}{|c|}{$0.26(0.8 I)$} & 0.75 & \multicolumn{2}{|c|}{$1.29(0.26 / 6.35)$} & $-0.19(0.54)$ & \multicolumn{2}{|c|}{0.73} & $-1.25 / 0.87$ \\
\hline Time & \multicolumn{2}{|c|}{$-0.18(0.05)$} & 0.00 & \multicolumn{2}{|c|}{$0.84(0.75 / 0.93)$} & $-0.14(0.04)$ & \multicolumn{2}{|l|}{0.00} & $-0.22 /-0.06$ \\
\hline SUD & \multicolumn{2}{|c|}{$0.81(0.35)$} & 0.02 & \multicolumn{2}{|c|}{$2.25(1.14 / 4.43)$} & $0.84(0.25)$ & \multicolumn{2}{|l|}{0.00} & $0.36 / 1.33$ \\
\hline Sex & \multicolumn{2}{|c|}{$0.87(0.37)$} & 0.02 & \multicolumn{2}{|c|}{$2.39(1.16 / 4.92)$} & $0.59(0.30)$ & \multicolumn{2}{|l|}{0.05} & $0.01 / 1.17$ \\
\hline Age & \multicolumn{2}{|c|}{$-0.04(0.02)$} & 0.13 & \multicolumn{2}{|c|}{$0.97(0.92 / 1.01)$} & $-0.02(0.02)$ & \multicolumn{2}{|l|}{0.37} & $-0.05 / 0.02$ \\
\hline Time:SUD & \multicolumn{2}{|c|}{$0.03(0.11)$} & 0.81 & \multicolumn{2}{|c|}{ I.03 (0.82/I.29) } & $0.00(0.10)$ & \multicolumn{2}{|l|}{0.97} & $-0.19 / 0.19$ \\
\hline
\end{tabular}

Note: B (eta), estimate.

Abbreviations: SE, standard error; $P, P$-value; OR, odds ratio; $\mathrm{Cl}$, confidence interval; SUD, substance use disorders; Time:SUD, time $\times$ SUD interaction; ER, emergency room. 

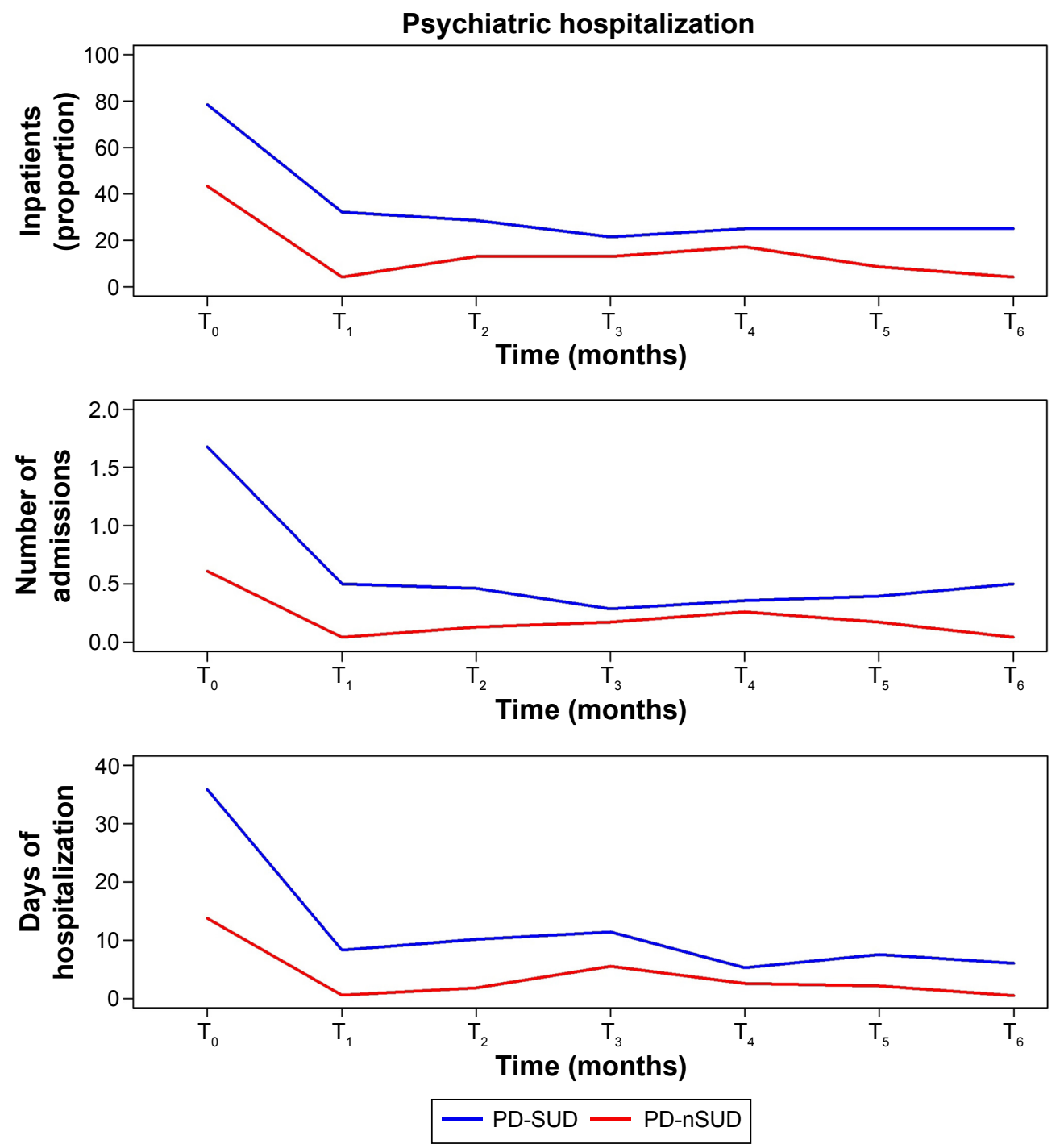

Figure 2 Changes in psychiatric hospitalizations (36 months).

Notes: $T_{0}$ : it includes the 6 months before treatment start; $T_{1}$ : it includes the 6 months of treatment; $T_{2}:$ months $7-12$ from treatment start; $T_{3}$ : months I3-18; $T_{4}$ : months 19-24; $T_{5}$ : months $25-30 ; T_{6}$ : months $31-36$.

Abbreviations: PD-SUD, personality disorder with comorbid substance use disorder; PD-nSUD, personality disorder without substance use disorder.

effective techniques borrowed from therapeutic models including both DBT and MBT. Our findings indicate that this treatment approach significantly reduced psychiatric inpatient readmissions and repeated psychiatric ER visits in patients with comorbid SUD-PD and in patients with PD alone. Importantly, these benefits were achieved in a sample of patients with severe PD (primarily BPD) who had poor general functioning and a high prevalence of suicide attempts and self-harm behaviors. In addition, these gains were maintained during the 36-month follow-up. These findings suggest that PD patients with concurrent SUD should not be excluded from psychotherapeutic interventions given that treatment outcomes in this group are equally as good as in patients with $\mathrm{PD}$ alone.
Based on the available evidence, ${ }^{2,44}$ PD-SUD patients are younger, have lower levels of education, are less likely to be married, have more severe psychopathology, and are more impulsive than patients with PD-nSUD. However, in our study, although the PD-SUD group was younger, differences in other sociodemographic characteristics were not significantly different from the PD-nSUD group. Not surprisingly, PD-SUD patients had received more previous psychiatric inpatient treatment, which has been associated with the PD severity in patients admitted for treatment, ${ }^{19,23,27}$ presented more crisis events, and also had a lower GAF score. Objective severe symptoms (ie, suicidal and self-harming behaviors), together with (as in other studies ${ }^{19,20,22,24,45}$ ) data on the patient's overall level of functioning (GAF), provide 

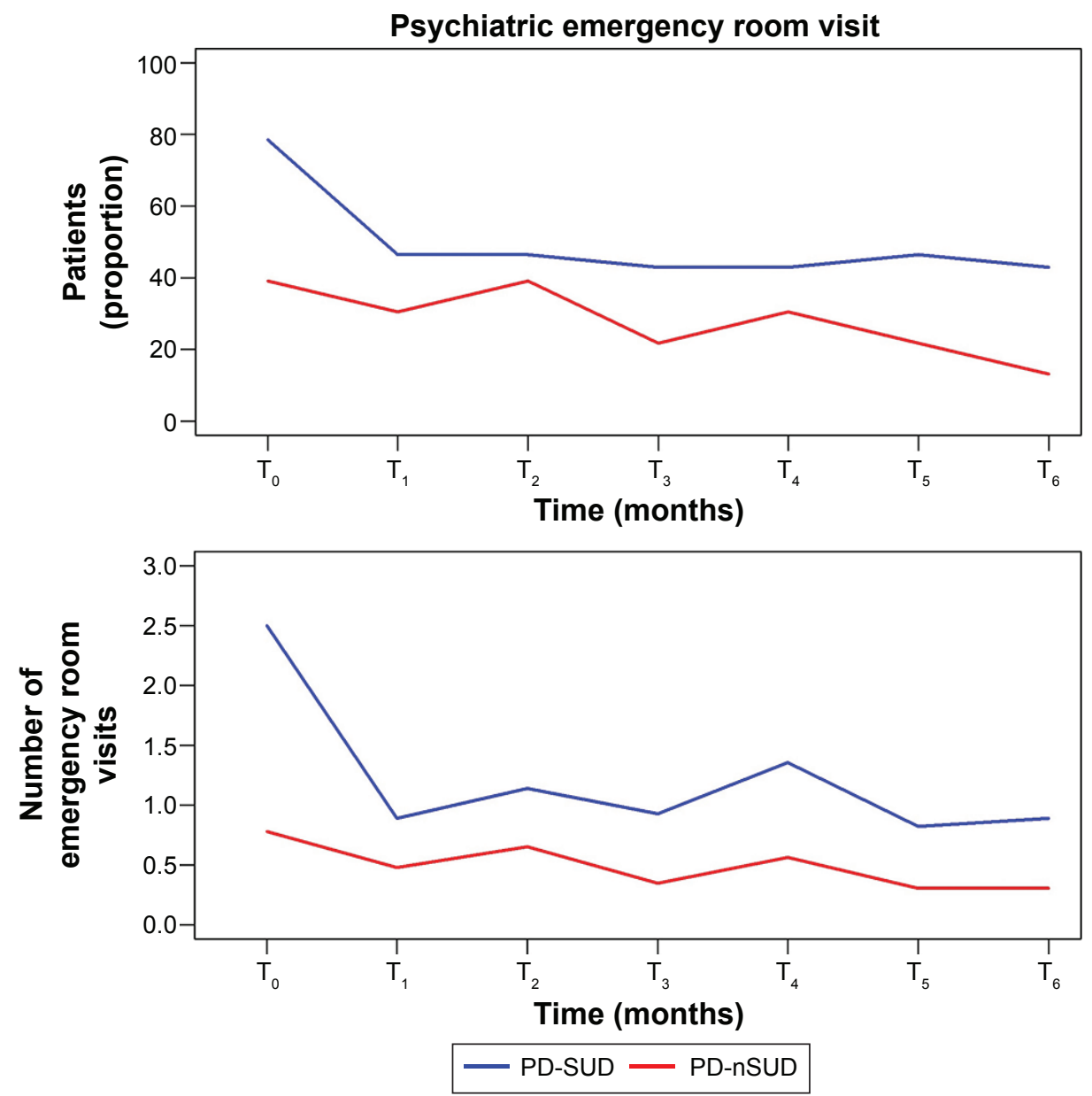

Figure 3 Changes in psychiatric emergency room visit (36 months).

Notes: $T_{0}$ : it includes the 6 months before treatment start; $T_{1}$ : it includes the 6 months of treatment; $T_{2}:$ months $7-12$ from treatment start; $T_{3}:$ months I3-18; $T_{4}$ : months 19-24; $T_{5}$ : months 25-30; $T_{6}$ : months 31-36.

Abbreviations: PD-SUD, personality disorder with comorbid substance use disorder; PD-nSUD, personality disorder without substance use disorder.

a longitudinal and comprehensive clinical assessment in patients characterized by high levels of impulsivity and instability whose symptoms could change in a matter of hours. Although the PD-SUD patients presented more behavioral disorders and transient psychotic symptoms, these differences were not significant, probably because of the high proportion of patients in both groups with BPD. Nevertheless, the baseline prevalence of self-aggressive and heteroaggressive behavior and transient psychotic symptoms in the PD-SUD group in this study was among the highest reported in studies that have assessed the efficacy of psychotherapeutic interventions for PD. ${ }^{17-26,29,30,33,35}$ Given that our sample consisted of patients with severe disorders (with low levels of global functioning), the good results in terms of efficacy of our psychotherapeutic approach cannot be attributed to low-severity disorders.

The main conclusion of the study is that specialized psychotherapy for severe PD can be effectively applied to
PD patients with concurrent SUD. Importantly, our findings suggest that the treatment effect size may be higher in patients with dual disorders compared to PD patients who do not suffer from SUD. These findings have important clinical implications, as they strongly suggest that the traditional practice of excluding these dual patients from treatment in specialized psychotherapy studies does not seem justified and may, therefore, reduce the external validity of such studies because of sample bias. Furthermore, these findings indicate that evidence-based psychotherapy approaches for severe PD should be made available to PD-SUD patients as part of the usual mental health clinical care practice. Unlike many studies, we did not exclude any of the PD-SUD patients during the assessment process.

We believe that the good results in terms of accessibility described in our study can be explained, at least in part, by specific management and clinical factors. Prior to launching the program, an informational session about the study 
objectives was held in each of the three mental health centers that serve the community area. During this meeting, inclusion and exclusion criteria were thoroughly discussed, and we clearly specified that the goal of the program was to make the program as accessible as possible (although we also explained the circumstances under which treatment was not appropriate). The flow of participants through the study (Figure 1) showed that 14 of patients referred for treatment did not attend any assessment interview and were therefore not admitted to treatment. We do not have accurate information about the percentage of nonattending patients who also had concurrent SUD because the only information available was the referral report form. However, several authors ${ }^{7,14,46}$ have pointed out the difficulties in engaging the more disturbed SUD patients in structured therapeutic programs. Therefore, one possible hypothesis for why we did not exclude from the study any of the PD-SUD patients could be that the referring physicians accurately described the treatment program and this may have dissuaded the more behaviorally disturbed SUD patients from attending the assessment interview.

Our results are consistent with the findings previously reported in other studies. Two studies ${ }^{33,35}$ showed that standard DBT can be effectively applied in patients with BPD and comorbid SUD (although it should be noted that these studies included only female patients). However, in contrast to our study, those two studies did not evaluate psychiatric hospitalizations and ER visits - both of which are well-known pragmatic variables that often reveal worse outcomes in SUD patients. ${ }^{46}$

The results of our study at 36 months also seem to indicate that the beneficial effects of this treatment may be longlasting, even in patients with severe disorders. Moreover, these benefits were confirmed by objective outcome measures rather than subjective measures. Obviously, sustained recovery in our group cannot be attributed solely to the PD intervention, but it seems likely that standard psychiatric treatment after completion of this program would be more effective than before. ${ }^{27}$

This study has several limitations. The main limitation is the lack of a control group. As a result, the outcomes reported here may only show the course of PD in this treatment setting. However, the results of the only RCT with long-term follow-up conducted at a day hospital in a PD sample whose severity and psychosocial functioning (mean GAF $\leq 40$ ) was similar to that of our study $y^{23,24,45}$ found that the need for psychiatric hospitalization in patients who followed treatment as usual did not improve gradually over time, rather it worsened and was highly irregular. Thus, the proportion of psychiatric inpatient admissions in patients under treatment as usual 24,30 , and 36 months after treatment initiation was $36.8 \%$, $36.8 \%$, and $73.7 \%$, respectively. ${ }^{47}$ Another limitation of our study is related to difficulties in reliably monitoring suicide attempts and substance use throughout the entire 36-month follow-up, as resource limitations made this unfeasible. However, the frequency of hospitalization episodes and ER visits is a reasonable approximation of serious suicidal behavior and severe self-harm acts. ${ }^{19,45}$ In fact, patients are offered hospitalization in anticipation of suicide attempts and severe self-harm, ${ }^{19}$ which, in many cases, would prevent these behaviors. Nevertheless, a reduction in hospitalizations and ER visits does not assure an overall clinical improvement and cannot in any way be considered the only purpose of severe PD patient treatment. However, this is a rational approach that allows a pragmatic assessment of clinical progress or worsening of severe PD. Finally, the relatively small sample size of this study is a limitation, although it is worth mentioning that the sample size was comparable to one of the other studies in the literature that assessed the efficacy of psychotherapeutic interventions for PD. ${ }^{16,32}$

In contrast to the aforementioned limitations, our study also has several important strengths related to its pragmatic design, treatment accessibility for SUD patients, the use of objective outcome measures (hospitalizations and ER visits), and the long ( 3 years) follow-up period. In this sense, the Cochrane Review ${ }^{16}$ has pointed out that it is necessary to replicate the results of RCTs of BPD in "real-world" studies. Likewise, several authors ${ }^{16,24,31,32}$ have argued that pragmatic studies are needed in severe PD to determine whether the treatment efficacy found in controlled studies can be replicated under usual practice conditions, and this is what we have done in the present pragmatic study. ${ }^{32,48}$ Another strength of our study is that data were collected as part of the usual mental health care routine, a fact that further reinforces the external validity of the study.

Despite the good results of this study, much more research is needed to develop specialized programs tailored for this difficult-to-treat population, and such programs should be implemented according to therapeutic strategies based on evidence-based interventions. ${ }^{49}$ It might be beneficial to consider programs designed for patients with $\mathrm{PD}$ and specific SUD (eg, PD + alcohol, cocaine, or opiate use disorder). ${ }^{50}$

\section{Conclusion}

The results of this study demonstrate that, under usual practice conditions, specialized psychotherapy for severe PD can be effectively applied in PD patients with comorbid SUD 
and that the benefits of this intervention can last for long periods of time (36 months in this study). These results are consistent with, and thus provide further support for, previously reported findings. The available data from this study and others strongly suggest that patients with dual PD-SUD should not be excluded from specialized psychotherapy studies. Moreover, PD-SUD patients should have access to treatment approaches such as the one used in this study as part of the usual mental health care routine.

\section{Acknowledgment}

The authors thank Bradley Londres for his invaluable assistance in editing the manuscript.

\section{Author contributions}

FL conceived and designed the study. FL, CSG, and JMB managed the data collection. NDA and FL performed the data analyses. FL, NDA, and JMB reported the study results. All authors contributed to the background, analysis of data, and drafting and revision of the manuscript. All authors have read and approved the final version of the manuscript.

\section{Disclosure}

The authors report no conflicts of interest in this work.

\section{References}

1. Grant BF, Stinson FS, Dawson DA, Chou SP, Ruan WJ, Pickering RP. Co-occurrence of 12-month alcohol and drug use disorders and personality disorders in the United States: results from the National Epidemiologic Survey on Alcohol and Related Conditions. Arch Gen Psychiatry. 2004;61(4):361-368.

2. Langås MA, Malt UF, Opjordsmoen S. In-depth study of personality disorders in first-admission patients with substance use disorders. $B M C$ Psychiatry. 2012;12:180.

3. Skodol AE, Oldham JM, Gallaher PE. Axis II comorbidity of substance use disorders among patients referred for treatment of personality disorders. Am J Psychiatry. 1999;156(5):733-738.

4. Trull TJ, Sher KJ, Minks-Brown C, Durbin J, Burr R. Borderline personality disorder and substance use disorders: a review and integration. Clin Psychol Rev. 2000;20(2):235-253.

5. Lee JH, Bagge CL, Schumacher JA, Coffey SF. Does comorbid substance use disorder exacerbate borderline personality features? A comparison of borderline personality disorder individuals with vs without current substance dependence. Personal Disord. 2010;1(4): 239-249.

6. Links PS, Heslegrave RJ, Mitton JE, van Reekum R, Patrick J. Borderline personality disorder and substance abuse: consequences of comorbidity. Can J Psychiatry. 1995;40(1):9-14.

7. Lana F. Personality disorders and substance abuse disorders. Psychosocial strategies. Actas Esp Psiquiatr. 2001;29(1):58-66. Spanish.

8. Hasin D, Fenton MC, Skodol A, et al. Personality disorders and the 3-year course of alcohol, drug, and nicotine use disorders. Arch Gen Psychiatry. 2011;68(11):1158-1167.

9. Dimeff LA, Linehan MM. Dialectical behavior therapy for substance abusers. Addict Sci Clin Pract. 2008;4(2):39-47.

10. Pennay A, Cameron J, Reichert T, et al. A systematic review of interventions for co-occurring substance use disorder and borderline personality disorder. J Subst Abuse Treat. 2011;41(4):363-373.
11. Kienast T, Stoffers J, Bermpohl F, Lieb K. Borderline personality disorder and comorbid addiction. Dtsch Arztebl Int. 2014;111(16):280-286.

12. Ball SA, Maccarelli LM, LaPaglia DM, Ostrowski MJ. Randomized trial of dual-focused vs single-focused individual therapy for personality disorders and substance dependence. J Nerv Ment Dis. 2011;199(5): 319-328.

13. Lee CW, Arntz A. A commentary on the study on dual-focused vs single-focused therapy for personality disorders and substance dependence by Ball et al (2011): what can we really conclude? J Nerv Ment Dis. 2013;201(8):712-713.

14. Karterud S, Arefjord N, Andresen NE, Pedersen G. Substance use disorders among personality disordered patients admitted for day hospital treatment. Implications for service developments. Nord J Psychiatry. 2009;63(1):57-63.

15. Bateman A, Fonagy P. Effectiveness of psychotherapeutic treatment of personality disorders. Br J Psychiatry. 2000;177(2):138-143.

16. Stoffers JM, Völlm BA, Rücker G, Timmer A, Huband N, Lieb K. Psychological therapies for people with borderline personality disorder. Cochrane Database Syst Rev. 2012;8:CD005652.

17. Linehan MM, Comtois KA, Murray AM, et al. Two-year randomized controlled trial and follow-up of dialectical behavior therapy vs therapy by experts for suicidal behaviors and borderline personality disorders. Arch Gen Psychiatry. 2006;63(7):757-766.

18. Giesen-Bloo J, van Dyck R, Spinhoven P, et al. Outpatient psychotherapy for borderline personality disorder. Randomized trial of schema focused therapy vs transference focused psychotherapy. Arch Gen Psychiatry. 2006;63(6):649-658.

19. Bateman A, Fonagy P. Randomized controlled trial of outpatient mentalization-based treatment versus structured clinical management for borderline personality disorder. Am J Psychiatry. 2009;166(12):1355-1364.

20. Doering S, Hörz S, Rentrop M, et al. Transference-focused psychotherapy vs treatment by community psychotherapists for borderline personality disorder: randomised controlled trial. Br J Psychiatry. 2010; 196(6):389-395.

21. McMain SF, Guimond T, Streiner DL, Cardish RJ, Links PS. Dialectical behavior therapy compared with general psychiatric management for borderline personality disorder: clinical outcomes and functioning over a 2-year follow-up. Am J Psychiatry. 2012;169(6):650-661.

22. Wilberg T, Urnes O, Friis S, Irion T, Pedersen G, Karterud S. One year follow-up of day treatment for poorly functioning patients with personality disorders. Psychiatr Serv. 1999;50(10):1326-1330.

23. Bateman A, Fonagy P. Effectiveness of partial hospitalization in the treatment of borderline personality disorder: a randomized controlled trial. Am J Psychiatry. 1999;156(10):1563-1569.

24. Karterud S, Pedersen G, Bjordal, et al. Day treatment of patients with personality disorders: experiences from a Norwegian treatment research network. J Pers Disord. 2003;17(3):243-262.

25. Yen S, Johnson J, Costello E, Simpson EB. A 5 day dialectical behavior therapy partial hospital program for women with borderline personality disorder: predictors of outcome from a 3-month follow-up study. J Psychiatr Pract. 2009;15(3):173-182.

26. Bales D, van Beek N, Smits M, et al. Treatment outcome of 18 month day hospital mentalization based treatment (MBT) in patients with severe borderline personality disorder in the Netherlands. J Pers Disord. 2012; 26(4):568-582.

27. Lana F, Sánchez-Gil C, Ferrer L, et al. Effectiveness of an integrated treatment for severe personality disorders. A 36-month pragmatic follow-up. Rev Psiquiatr Salud Ment. 2015;8(1):3-10.

28. Gabbard GO, Coyne L, Allen JG, Spohn H, Colson DB, Vary M. Evaluation of intensive inpatient treatment of patients with severe personality disorders. Psychiatr Serv. 2000;51(7):893-898.

29. Linehan MM, Armstrong HE, Suarez A, Allmon D, Heard HL. Cognitive-behavioral treatment of chronically parasuicidal borderline patients. Arch Gen Psychiatry. 1991;48(12):1060-1064.

30. Verheul R, Van Den Bosch LMC, Koeter MW, De Ridder MAJ, Stijnen T, Van Den Brink W. Dialectical behaviour therapy for women with borderline personality disorder: 12 month, randomised clinical trial in The Netherlands. Br J Psychiatry. 2003;182(2):135-140. 
31. Hoertel N, López S, Wang S, González-Pinto A, Limosin F, Blanco C. Generalizability of pharmacological and psychotherapy clinical trial results for borderline personality disorder to community samples. Personal Disord. 2015;6(1):81-88.

32. Lana F, Fernández San Martin MI. To what extent are specific psychotherapies for borderline personality disorders efficacious? A systematic review of published randomised controlled trials. Actas Esp Psiquiatr. 2013;41(4):242-252.

33. Van den Bosch LM, Verheul R, Schippers GM, Van den Brink W. Dialectical behavior therapy of borderline patients with and without substance use problems. Implementation and long-term effects. Addict Behav. 2002;27(6):911-923.

34. Bornovalova MA, Daughters SB. How does dialectical behavior therapy facilitate treatment retention among individuals with comorbid borderline personality disorder and substance use disorders? Clin Psychol Rev. 2007;27(8):923-943.

35. Harned MS, Chapman AL, Dexter-Mazza ET, Murray A, Comtois KA, Linehan MM. Treating cooccurring Axis I disorders in recurrently suicidal women with borderline personality disorder: a 2-year randomized trial of dialectical behavior therapy versus community treatment by experts. J Consult Clin Psychol. 2008;76(6):1068-1075.

36. American Psychiatric Association. Diagnostic and Statistical Manual of Mental Disorders. 4th ed. Washington, DC: APA; 1994.

37. First MB, Spitzer RL, Gibbon M, Williams JB, Smith L. Entrevista clínica estructurada para los trastornos de la personalidad del eje II del DSM-IV. SCID-II. Barcelona, Spain: Masson; 1999.

38. Livesley WJ. A practical approach to the treatment of patients with borderline personality disorder. Psychiatr Clin North Am. 2000;23(1): 211-232.

39. Linehan, M. Cognitive-Behavioral Treatment of Borderline Personality Disorders. New York, NY: The Guilford Press; 1993.
40. Bateman A, Fonagy P. Mentalization-Based Treatment for Borderline Personality Disorders. Oxford, UK: Oxford University Press; 2006.

41. American Psychiatric Association Practice Guidelines. Practice guideline for the treatment of patients with borderline personality disorder. Am J Psychiatry. 2001;158(10 Suppl):1-52.

42. Durlak AD. How to select, calculate, and interpret effect sizes. J Pediatr Psychol. 2009;34(9):917-928.

43. Liang KY, Zeger SL. Longitudinal data analysis using generalized linear models. Biometrika. 1986;73(1):13-22.

44. Wapp M, van de Glind G, van Emmerik-van Oortmerssen K, et al; IASP Research Group. Risk factors for borderline personality disorder in treatment seeking patients with a substance use disorder: an International Multicenter Study. Eur Addict Res. 2015;21(4):188-194.

45. Bateman A, Fonagy P. 8-year follow-up of patients treated for borderline personality disorder: mentalization-based treatment versus treatment as usual. Am J Psychiatry. 2008;165(5):631-638.

46. Young MM, Jesseman RJ. The Impact of Substance Use Disorders on Hospital Use. Ottawa, ON: Canadian Centre on Substance Abuse; 2014.

47. Bateman A, Fonagy P. Treatment of borderline personality disorder with psychoanalytically oriented partial hospitalization: an 18-month follow-up. Am J Psychiatry. 2001;158(1):36-42.

48. Paris J. Stepped care an alternative to routine extended treatment for patients with borderline personality disorder. Psychiatr Serv. 2013;64(10) $1035-1037$.

49. Livesley WJ. An integrated approach to the treatment of personality disorder. J Ment Health. 2007;16(1):131-148.

50. Carpenter RW, Wood PK, Trull TJ. Comorbidity of borderline personality disorder and lifetime substance use disorders in a nationally representative sample. J Pers Disord. 2015;20(4):1-15.
Neuropsychiatric Disease and Treatment

\section{Publish your work in this journal}

Neuropsychiatric Disease and Treatment is an international, peerreviewed journal of clinical therapeutics and pharmacology focusing on concise rapid reporting of clinical or pre-clinical studies on a range of neuropsychiatric and neurological disorders. This journal is indexed on PubMed Central, the 'PsycINFO' database and CAS,

\section{Dovepress}

and is the official journal of The International Neuropsychiatric Association (INA). The manuscript management system is completely online and includes a very quick and fair peer-review system, which is all easy to use. Visit http://www.dovepress.com/testimonials.php to read real quotes from published authors. 